\title{
Communication et changement dans les organisations
}

Nicole Giroux

\section{(2) OpenEdition}

1 Journals

Édition électronique

URL : http://journals.openedition.org/communicationorganisation/1615

DOI : 10.4000/communicationorganisation. 1615

ISSN : $1775-3546$

Éditeur

Presses universitaires de Bordeaux

Édition imprimée

Date de publication : 1 mai 1993

ISSN : 1168-5549

Référence électronique

Nicole Giroux, "Communication et changement dans les organisations », Communication et organisation [En ligne], 3| 1993, mis en ligne le 26 mars 2012, consulté le 30 avril 2019. URL : http:// journals.openedition.org/communicationorganisation/1615; DOI : 10.4000/ communicationorganisation. 1615

Ce document a été généré automatiquement le 30 avril 2019.

(c) Presses universitaires de Bordeaux 


\title{
Communication et changement dans les organisations
}

\author{
Nicole Giroux
}

1 Ce dossier vous convie à explorer la relation entre la communication et le changement au sein des organisations. Si ce thème a été ainsi mis à l'ordre du jour par l'équipe québécoise responsable de ce dossier, c'est qu'il nous est apparu prioritaire dans le champ des communications organisationnelles. En effet, des transformations majeures tant technologiques que culturelles, économiques et politiques remettent aujourd'hui en question la structure et le fonctionnement de bon nombre d'organisations. Confrontées aux défis de la mondialisation des marchés, de l'interculturalisme, de l'informatisation, de la constitution de nouvelles entités politiques et économiques, les organisations doivent s'adapter à ce nouveau contexte dont les règles du jeu sont encore en définition.

2 Cette turbulence de l'environnement n'est pas nouvelle. Elle a déjà été soulignée par Emery et Trist (1965) mais elle semble augmenter (McCann et Selsky, 1984) tant par son intensité que par son ampleur. Les systèmes humains étant de plus en plus interconnectés, les variations et les perturbations s'y propagent plus rapidement. Il n'est donc pas surprenant de constater que le changement a fait l'objet de nombreuses études et publications en sciences administratives, qu'il est devenu un produit vedette des firmes de consultants et une préoccupation constante des praticiens.

3 L'expérience devait toutefois montrer aux chercheurs, comme aux consultants et aux praticiens que le changement est souvent difficile, long et coûteux et qu'il est tout aussi malaisé de définir son contenu que son processus.

4 Les échecs dans les tentatives de changement - et ils sont nombreux - ont souvent été attribués à la résistance au changement et à des problèmes de communication. Le problème résiderait alors dans les personnes et dans les modes d'interaction entre cellesci.

5 Lorsque l'on conçoit l'organisation comme un tissu de communication, un système de transactions (Taylor, 1988, 1992) et comme un processus organisant composé de comportements interconnectés (Weick, 1979), il devient évident que le changement organisationnel est, par définition, délicat. Changer l'organisation, c'est changer les 
comportements des personnes et leurs relations entre elles. C'est du même coup changer leur position dans des réseaux d'engagement, leur contexte de création de signification. C'est toucher à leur identité, à leur quotidienneté. Dans cette perspective, la résistance au changement et les problèmes de communication ne sont plus considérés comme des barrières au changement mais bien comme des produits naturels du changement.

Malheureusement, la résistance au changement et les problèmes de communication sont trop souvent perçus de façon négative, comme des ennemis à éliminer. On tente alors de faire disparaitre la résistance par la persuasion, la coercition ou par l'exclusion des récalcitrants. On essaie de remédier aux problèmes de communication par plus de communication verticale descendante qui prend souvent la forme de décret, d'exhortation ou d'endoctrinement.

7 Ces façons de faire, souvent intempestives et contreproductives (Beer, 1990), ne sont pas le fruit de mauvaises intentions de la part des agents de changement. Elles résultent plutôt des carences de nos modèles de l'organisation, du changement et de la communication.

8 Les agents de changement qu'ils soient dirigeants ou consultants, sont contraints par des impératifs de temps et de coûts. Conscients de leurs propres limites et forts de leur expérience de la subtilité de la gestion des personnes, ils interrogent sans cesse les théoriciens pour améliorer leur performance. Leurs questions portent, le plus souvent, sur le «comment faire le changement?» Cette question est très légitime et fort pertinente. En fait, ils soulèvent la question fondamentale de la gestion des processus organisant et désorganisant puisque changer c'est tout autant détruire un ordre ancien que construire un ordre nouveau (Woolsey Biggart, 1977; Gray, Bougon et Donnellon, 1985). Hélas! cette question intelligente reçoit trop souvent une réponse simpliste. On croit que les agents de changement recherchent une recette facile alors qu'ils souhaitent mieux comprendre la complexité de leur organisation.

9 Les modèles qu'on leur offre sont issus d'une théorie fonctionnaliste de l'organisation qui décrit celle-ci comme une machine plus ou moins sophistiquée (Morgan, 1986). Dans cette perspective, le dirigeant est présenté comme un démiurge, qui appuyant sur les manettes structure, technologie et culture, transformera, comme par magie, son organisation. Dans cette optique, la communication est vue comme un conduit (Axley, 1984). Il suffit pour le dirigeant de bien encoder son message, de choisir les médias de communication appropriés pour que sa «bonne parole» soit reçue, comprise et incarnée dans de nouveaux comportements.

10 Cette vision simplifie considérablement la complexité de l'organisation. Elle considère le dirigeant comme un macro-acteur utilisant des macro-outils pour façonner un groupe humain vivant et dynamique. Cette approche, bien que réductrice, est néanmoins utile. Elle souligne que le dirigeant, responsable de l'organisation, jouit, de par sa position, d'une influence considérable sur la communication et le changement (Peters 1978). Ainsi, celui-ci, placé au sommet de l'organisation et à l'interface de l'environnement, occupe une position privilégiée dans les réseaux de communication. Il est à même de développer une vision globale et stratégique de son organisation. Il bénéficie, de plus, d'une autorité formelle qui donne du poids à son discours. Il contrôle des ressources qui lui confèrent un pouvoir considérable.

11 Cette approche montre que le dirigeant peut influencer: 1) le contexte de vie de ses subordonnes en répartissant les tâches et les responsabilités (action sur la structure); 
2)leurs expériences et les stimuli qu'ils reçoivent et donc les données à partir desquelles ils généreront du sens (action sur la culture) ; 3) leur milieu physique, leur relation au travail et entre eux (action sur les technologies de production et de communication). Néanmoins, elle ne nous dit pas comment ces macro-actions se traduisent en nouveaux comportements. Elle indique les instruments à utiliser mais sans nous donner vraiment le mode d'emploi !

12 L'approche fonctionnaliste minimise aussi le fait que le dirigeant est aussi un microacteur, limité dans ses capacités cognitives et communicationnelles. Elle passe sous silence une dimension fondamentale de la gestion, à savoir la dépendance du dirigeant par rapport à ses subordonnés. Gérer c'est faire en sorte que des individus ayant des capacités, des intérêts et des aspirations différentes, coopèrent à la réalisation d'une œuvre collective (Barnard, 1938). Le dirigeant ne peut donc à lui seul faire le changement. Il a besoin de la coopération des autres membres de l'organisation qui, bien que ne possédant pas autant de ressources que lui, bénéficient tout de même d'une marge de manœuvre (Crozier et Friedberg, 1977), d'une capacité à agir, à construire du sens et à communiquer. Il a besoin, en somme, de la collaboration des micro-acteurs de l'organisation, de leur engagement dans le changement. Or, on sait peu de chose sur les processus par lesquels se construit cet engagement.

13 La perspective fonctionnaliste met en lumière la dimension macro de l'organisation qu'elle présente comme une entité. Il s'agit donc d'une vision globalisante. La dimension micro de l'organisation est, quant à elle, soulignée par l'approche des processus organisants qui focalise sur les interactions locales. Ces deux points de vue, que l'on oppose le plus souvent, sont complémentaires. Le grand défi d'une théorie renouvelée de l'organisation est la construction d'un modèle permettant d'articuler le macro et le micro, de comprendre à la fois la communication dans sa quotidienneté au sein de l'organisation et dans son expression à travers le discours organisationnel.

14 Il faut, somme toute, comprendre comment relier le changement global et le changement local. Cela suppose d'étudier le changement non seulement tel que conçu par le dirigeant au sommet mais aussi tel que vécu et approprié par les autres membres de l'organisation. Cela suppose d'analyser la communication non seulement comme un système ou un réseau construit mais aussi comme une performance inscrite dans l'instant (Pacanowski et Trujillo, 1982), un processus qui s'étale dans le temps (Hawes, 1973, 1974) une chaîne d'interactions, porteuse de légitimité, génératrice d'énergie et d'engagement (Collins, 1981, 1987 ; Kemper et Collins, 1990 ;Westley, 1990).

15 Nous avons donc besoin de modèles de l'organisation, du changement et de la communication qui tiennent compte de la diversité interne de l'entreprise, de la dimension processuelle et contingente de l'action collective, du caractère moral des transactions de communication réalisées en contexte organisationnel.

Ce dossier thématique s'inscrit dans cette démarche. Il propose des textes théoriques et empiriques visant à une reconceptualisation de l'organisation et de la communication. Il décrit le rôle de la communication dans différents processus de changement. Il tente de montrer les liens entre différents niveaux de changement (sociétal, industriel, organisationnel, individuel) et de relier ainsi la perspective macro et la perspective micro.

17 Lee Thayer, un pionnier de la communication organisationnelle, nous livre ses réflexions sur la vie des organisations. Après avoir posé le caractère indissociable de la communication et de l'organisation, il montre les limites de nos modèles actuels. Il nous 
invite à réfléchir sur la finalité des organisations et à développer des théories de l'organisation et de la communication orientées vers l'épanouissement des êtres humains.

James Taylor ouvre une avenue en ce sens. Il propose une théorie communicationnelle de l'organisation présentant celle-ci comme un discours. Ce discours s'exprime selon deux modalités qui sont en tension: la conversation et le texte. Il fait ressortir le caractère fondamental des transactions productrices de stabilité et de changement. Il présente trois modèles de changement : le changement qui émane du texte, le changement généré par la conversation et le changement interactif du texte et de la conversation.

Rene Bouwen et Ronald Fry s'intéressent aux processus d'innovation et d'apprentissage dans les organisations. À partir d'une étude empirique, ils décrivent quatre types de dialogues entre la logique dominante et la logique nouvelle durant le changement. Ils montrent que le développement d'organisations « apprenantes » (learning organizations) est facilité par la présence d'un discours interne réflexif basé sur la confrontation et la recherche d'un consensus.

Christiane Demers examine le processus de diffusion du changement radical dans une organisation complexe. Dans son approche, elle intègre les processus de transmission et d'interprétation en montrant la perspective des dirigeants et celle des autres membres de l'organisation. Elle modélise le changement selon deux dynamiques (verticale et horizontale) dont l'interaction peut entraîner soit l'intégration soit la politisation de l'organisation.

21 Diane Parent, dans une démarche interprétative, montre comment les transformations structurelles de l'industrie agricole influencent les représentations des membres des entreprises familiales. Elle décrit l'impact de ces transformations sur les valeurs et l'identité des personnes concernées par la succession qui devient alors un enjeu important.

Guy Cameron, un praticien de la communication organisationnelle, expose, dans la rubrique Expériences, l'influence $\mathrm{du}$ contenu et du contexte sur le processus de changement. Analysant son organisation, il souligne l'importance du leadership au sommet et de la crédibilité des agents locaux de changement. Il prône une pédagogie du changement adaptée aux caractéristiques de l'organisation et aux exigences temporelles de la démocratisation du changement.

Ces textes abordent tous le changement organisationnel dans une perspective communicationnelle. Ils ont en commun un souci de comprendre la dynamique quotidienne $\mathrm{du}$ changement telle que vécue par toutes les parties prenantes au changement et non plus du seul point de vue de la direction. Tous les auteurs soulignent, chacun à sa manière, la dimension morale, humaine, démocratique du changement. $\mathrm{Ce}$ faisant, ils dégagent des pistes de recherche et de réflexion pour l'étude de la relation entre communication et changement dans les organisations. Ils nous montrent surtout que la contribution des communicologues au changement est profondément humaniste. Elle vise à un partage, à une mise en commun du changement. En cela ils demeurent fidèles à la définition même de la communication. 


\section{BIBLIOGRAPHIE}

Axley, S. R., " Managerial and organizational Communication in terms of the Conduit Metaphor ", Academy of Management Review, 9, 428-437, 1984.

Barnard, C, The Function of the Executive, Cambridge, Harvard University Press, 1938, $334 \mathrm{p}$.

Beer, M., Eisenstat R. A. et Spector B., "Why Change Programs Don't Produce Change » Harvard Business Review, novembre et décembre, 158-166, 1990.

Collins, R., "On the Microfoundations of Macrosociology », American Journal of Sociology, 86/5, 984-1014, 1981.

Collins, Randall, «Interaction Ritual Chains, Power and Property: The Micro-Macro Connection as an Empirically Based Theoretical Problem » dans Alexander, Jeffery et al. The Micro-Macro-Link, University of California Press, 1987.

Crozier, Michel, Friedberg, Michel, L'acteur et le système, Seuil, Collection Points, Paris, 1977.

Emery, F. E. \& Trist, E., " The Causal Texture of Organizational Environments », Human Relations, 18, 21-31,1965.

Gray, B., M. G. Bougon, et A. Donnellon, « Organizations as Constructions and Destructions of Meaning ", Journal of Management, 11/2, 83-98, 1985

Hawes, L. G, "Social Collectivities As Communication », Quarterly Journal of Speech, 60, 497-502, 1974.

Hawes, L.C., "Elements of a Model for Communication Processes », Quarterly Journal of Speech, 59/1, 11-21, 1973.

Kemper, T. \& Collins R., « Dimensions of Microinteraction », American Journal of Sociology, 96/1, $32-68,1990$.

McCann, J. E. \& Selsky, J. «Hyperturbulence and the Emergence of Type 5 Environment », Academy of Management Review, 9 juillet, 101-128, 1984

McDaniel, J. B., Communication The Process of Organizing, Toronto, Allyn and Bacon, 403 p., 1977.

Morgan, G., Images of Organization, Beverley Hill, Sage, 1986.

Pacanowsky, M. E. et O'Donnell-Trujillo, N., « Communication and Organizational Cultures », Western Journal of Speech Communication, 42, 115-130,1982.

Peters, Thomas, J., « Symbols, Patterns and Settings : An Optimistic Case for Getting Things Done », Organizational Dynamics, Automne, 3-23, 1978.

Taylor, J.-R., Une organisation n'est qu'un tissu de communication, Université de Montréal, Cahiers de recherche en Communication, Montréal, 305 p.

Taylor, J. R., Rethinking the Theory of Organization, Ablex, sous presse.

Weick, K. E., The Social Psychology of Organizing, Addison Westley, Reading, MA, 1979

Westley, F., " Middle Managers and Strategy : Microdynamics of Inclusion ", Strategic Management Journal, 11, 337-351, 1990. 
Woolsey, B. N., "The Creative Destructive Process of Organizational Change: The Case of the Post Office ", Administrative Science Quarterly, 22, 410-425, 1977.

AUTEUR

NICOLE GIROUX

Département de communication, Université de Montréal 